WIENER SLAVISTISCHES JAHRBUCH, Band 57/2011, 77-83

(C) 2011 by Österreichische Akademie der Wissenschaften, Wien

GERTR A U DE Z A D

\title{
Dušan Hanáks Film „322“ als neo-avantgardistische Synthese der experimentellen Kunst der 1960er Jahre
}

Die experimentelle Literatur der 1960er Jahre kann in zwei grundlegende Richtungen eingeteilt werden. Jiří Holý etwa schreibt in seiner Geschichte der tschechischen Literatur des 20. Jahrhunderts von einer authentisch-dokumentarischen und von einer artifiziell-intellektuellen Prosa; als Beispiele nennt er interessanterweise Filmregisseure, nämlich im einen Fall Miloš Forman, Ivan Passer und Jaroslav Papoušek, im anderen Fall Jan Němec, Věra Chytilová und Juraj Jakubisko (vgl. Holý 2003: 286-291). ${ }^{1}$ Unter der Annahme, dass eine solche Zweiteilung nicht nur für die Literatur und nicht nur in der tschechischen, sondern auch in der slowakischen Kultur auszumachen ist, soll im Folgenden nachgewiesen werden, dass Hanáks Film die beiden, scheinbar gegensätzlichen Richtungen vereint und so als Synthese der experimentellen Kunst der sechziger Jahre erachtet werden kann.

Dušan Hanáks Film ,322“ wurde im Jahr 1968 gedreht. Er basiert auf einer Erzählung von Ján Johanides, die 1963 im Band Súkromie - dem Debüt des Autors erschienen ist und den Titel „Potápača prit’ahujú pramene mora“ trägt. Im Mittelpunkt dieser Novelle steht ein Ich-Erzähler, von dem man nicht viel mehr erfährt, als dass er Koch in einem Hotel ist, mittleren Alters, verheiratet und kinderlos. Seine Kontakte beschränken sich auf die nächste Umgebung: seine Frau, die im Lauf der Handlung bei einem Autounfall ums Leben kommt, und ihren Geliebten, der den Unfall heil übersteht, die Nachbarn, einen Kollegen am Arbeitsplatz, der ebenfalls betrogen wird, ein Küchenmädchen im Hotel, das ungewollt schwanger wird, und

${ }^{1}$ In der Lyrik hätte die erste Linie eine Entsprechung im existentialistischen Dichter Jan Zábrana oder im Beatnik Václav Hrabě, die zweite in der konkreten Poesie oder in der surrealistischen Lyrik - beide stehen für eine avantgardistische, material-orientierte Literatur, die mit der Konstruktion des Sprachmaterials, mit Spiel und Zufall, mit Elementen von Dada und Pop Art arbeitet. 
nicht zuletzt das Personal im Krankenhaus - der Held hat nämlich, das erfährt man gleich im ersten Absatz, Krebs.

Die Krankheit ist Mit-Ursache und Verstärker für das Lebensgefühl des Ich-Erzählers, das von einem grundsätzlichen Unbehagen in der Welt geprägt ist, von Fremdheit, Ratlosigkeit über die eigene Bestimmung, Bange und Einsamkeit. Schlüsselwörter im Text sind „smrt"“, „márnost"“, „,nepoznatel'nost"“, „hanba“, „,nevol'nost"“, ,únava“" und „nuda“; der Held versteht die Welt nicht und wird auch nicht verstanden: „,nechápal som“, ,,nerozumel som“, heißt es wiederholt, „zdalo sa mi to všetko zbytočné“ und „nechápal by moje slova“.

Der Novellenband Súkromie entstand unter dem Einfluss der existentialistischen Philosophie von Jean-Paul Sartre und Albert Camus. Im Jahr 1963 kam es einer Sensation gleich, dass Johanides seine Aufmerksamkeit von der Außenwelt auf das Innere des Menschen richtet, vom Kollektiv auf das Individuum und seine Befindlichkeiten. Statt eines, so der zeitgenössische Kritiker Jozef Felix, „,̌lovek-schéma, človek-formula“, wie man ihn in der Literatur der fünfziger Jahre findet, stellt der Autor einen ,človek trpiaci, zápasiaci, bezmocný“ in den Mittelpunkt seiner Geschichte (Felix 1997: 186). Er tritt damit aus der vermeintlich heilen Welt des Sozialismus in eine absurde, sich auflösende Welt (vgl. Felix 1997: 185), in der das Geschehen bloß festgestellt, aber nicht mehr gedeutet oder bewertet wird. Erzähltechnisch greift Johanides die Neuerungen des französischen Nouveau roman auf und hält dem positivistischen Realismus seiner Zeit eine konkrete Sachlichkeit und diskursive Erzählweisen entgegen (vgl. Marčok 2004: 216).

Hanáks Film weicht in wichtigen Punkten von der literarischen Vorlage ab - beginnend mit dem Titel „322“, der die Nummer jener Krankheit bezeichnet, die beim Helden diagnostiziert wird. Die Grundstimmung ist wie in Johanides' Novelle ein existentialistisches Unbehagen in der Welt; der Film beschreibt aber nicht nur das subjektive Lebensgefühl des Helden, sondern dokumentiert darüber hinaus eine Reihe weiterer fragiler Existenzen und den Zustand der gesamten Gesellschaft, die ebenfalls von einer großen Unsicherheit geprägt ist. Die Geschichte des Helden wird im Film in einen größeren Kontext gestellt, sein Privatleben in einen gesellschaftlichen Rahmen eingebettet. Aus den verschiedenen Situationen, in denen sich die Protagonisten wiederfinden, entsteht das Panoptikum der kleinen Welt eines einfachen Menschen im sozialistischen Staat der ausgehenden sechziger Jahre, mitsamt ihrer Mode und Ästhetik.

Voraussetzung dafür ist eine erzähltechnische Abweichung vom Ausgangstext: die narrative Instanz des Ich-Erzählers wird im Film durch ein außenstehendes Kamera-Auge ersetzt. Der Regisseur bindet sich dadurch nicht mehr an den Standpunkt des Helden und sein subjektives Erleben. Indem die Kamera selbst wieder unterschiedliche Points of view einnimmt, öffnet sich der Raum für eine Vielfalt von Perspektiven, die die Vielfalt der Wirklichkeit und die Relativität der Wahrnehmung unterstreichen. Der Untertitel des Films, „zo života človeka, ktorý stratil vieru a ne- 
odvažuje sa neverit"“, 2 ist laut Hanák durchaus politisch gemeint: der Glaube an eine bessere Zukunft ist verloren gegangen, zurück bleibt die Ratlosigkeit.

Der Held im Film ist nicht nur Koch, sondern auch ehemaliger Parteifunktionär des Krajský národný výbor und hat in den fünfziger Jahren bei der Kollektivierung der Landwirtschaft mitgemacht. Er hat sich zwar aus der Politik zurückgezogen, ist aber trotzdem mit der Schuldfrage konfrontiert. Anders als die sogenannte Abrechnungsliteratur der sechziger Jahre, etwa Ludvík Vaculíks Roman Sekyra oder Milan Kunderas Žert ${ }^{3}$, ist Hanáks Film sehr vorsichtig mit Urteilen, die Schuldfrage bleibt wie viele andere Fragen im Film offen; der Held ist nicht Opfer, sondern ehemaliger Täter - aber trotzdem kein unsympathischer oder schlechter Mensch.

Auch die Krankenhaus- und Hotelszenen lassen keine Illusionen über die Mechanismen und Marasmen der planwirtschaftlichen Arbeitswelt und des Lebens unter kommunistischer Verwaltung aufkommen. Oft werden Missstände nur angedeutet - etwa wenn ein Blick aus dem Fenster eine Warteschlange zeigt, die sich vor einem Geschäft gebildet hat, oder wenn es den Helden auf die Suche nach dem Grab seiner Mutter in eine trostlose Stadt verschlägt, in der die Fassaden von den leerstehenden Gebäuden fallen. Das Thema wird aber nicht direkt verbalisiert, sondern nur durch das Bild aufgeworfen, das als eigener Bedeutungsträger fungiert.

„322“ war Hanáks erster abendfüllender Spielfilm - davor hatte er eine Reihe von Dokumentarfilmen gedreht, deren Techniken er auch in diesem Film einsetzt: eine natürliche Beleuchtung, die Handkamera, die eine gewisse Unruhe ins Bild bringt, und das Teleobjektiv, mit dem man auch bei schwierigen Lichtbedingungen nahe an das Geschehen herankommt. Die Milieus - die Stadt (gedreht wurde in Bratislava, Praha und Brno), das Hotel, das Krankenhaus, die Landschaft - sind ebenso real wie die Protagonisten realistisch; Hanák arbeitet auch mit Laiendarstellern. ${ }^{4}$ Manche Szenen sind, ähnlich wie die Filme von Ivan Passer oder die Prosa von Bohumil Hrabal, poetisch aufgeladen, etwa jene mit dem hässlichen, aber liebenswerten Küchenmädchen Cilka, das an Jarmilka, eine Figur aus Hrabals gleichnamiger Erzählung vom Anfang der fünfziger Jahre erinnert.

Authentisch, dokumentarisch, ungespielt, echt - das ist die eine Seite des Films. Auf der anderen Seite findet sich im Vorspann der Hinweis: „udalosti a motívy filmu sú vymyslené a nezhodujú sa so skutočnost’ou“, der insofern überflüssig ist, als der Film ohnehin keinen Zweifel daran lässt, dass es sich hier um eine künstliche Konstruktion handelt, um ein Spiel mit der Form und dem Material der literarischen Vorlage und des Films an sich.

2 Angeblich handelt es sich um ein Dostoevskij-Zitat, das jedoch bislang nicht nachgewiesen werden konnte.

3 Auch Kunderas Scherz wurde verfilmt, aber Jaromil Jirešs Film aus dem Jahr 1968 ist im Vergleich zu ,322“ relativ konventionell gemacht.

${ }^{4}$ Manche Gesichter, die man hier zu sehen bekommt, verweisen schon auf seinen nächsten Film, den Dokumentarfilm Obrazy starého světa aus dem Jahr 1972. 
Hanák wendet zahlreiche experimentelle filmtechnische Verfahren an. Was die Bilderfolge betrifft, sticht der häufige Wechsel der Einstellungen ins Auge, den der Drehbuch-Co-Autor Johanides heftig kritisierte: ${ }^{5}$ die Kamera verweilt nicht lange an einem Punkt, sondern ändert ihren Blickwinkel ständig, zum Beispiel von einer Person auf die andere: einmal sieht man die Frau im Auto aus der Position des Geliebten im Haustor, dann gleich wieder den Geliebten vom Volant aus; einmal sieht man das Paar auf Augenhöhe, dann durch den Zwischenraum zwischen dem Asphalt und den Rädern des vorbeifahrenden Zugs; einmal das durch die Landschaft fahrende Auto aus der Vogelperspektive, einmal von unten die oben sich drehenden Bäume und so weiter. Gerade in diesem Fall erinnert die bewegliche Kamera ein wenig an die Land Art der sechziger Jahre, die die Landschaft zum Objekt künstlerischer Gestaltung macht, und jedenfalls an Věra Chytilovás artifizielle Filmtechnik.

Experimentell ist auch die Verwendung der verschiedenen Medien und die Auflösung ihrer Grenzen. Zum Beispiel entsteht aus der Dynamik des Films, indem die laufenden Bilder angehalten werden, ein statisches Bild, eine Photographie. Manche Bilder sind durchkomponiert, manche verwenden auch die Protagonisten als Material. Erwähnenswert ist in diesem Zusammenhang auch eine Szene, in der eine Figur auf ein Bild zugeht, das an der Wand hängt, und dieses weitermalt, oder Szenen, aus denen sich Objekte ergeben, die dann isoliert dargestellt werden, etwa ein aus Zündhölzern zusammengebautes Rad. Auch die Graphik ist präsent - seien es die Typen der Schreibmaschine, mit der im Spital die Daten des kranken Helden aufgenommen werden, seien es die zitierten Zeitschriften und Bücher wie „Host do domu“ oder Hynek Alois Joukls Bildband Motylové a housenky středni Evropy, in denen einer der Protagonisten blättert. In manche Szenen werden Kapitelüberschriften eingeblendet. $^{6}$

Eine wichtige Rolle spielt darüber hinaus das Experiment mit dem Ton: Hanák setzt ihn häufig asynchron ein, das heißt, dass sich die Bild- und Tonsequenzen nicht decken, sondern dass der Ton weiterläuft, während das Bild schon wechselt, oder umgekehrt. Die Film-Musik reicht von einem Bach-Choral über Zymbalmusik und Cluster-Kompositionen bis hin zum Jazz; sie läuft meistens nicht als Begleitung und Bestätigung der Handlung, sondern als eigener Bedeutungsträger - oft gerade im Kontrast zum Bild. Es gibt auch musikalische Einlagen, zum Beispiel singt eine alte Frau im Restaurant den Schlager „Junge, komm bald wieder nach Haus ...“, in einer „krčma“ spielt slowakische Volksmusik und so weiter. Auch Tanz und Bewegung

${ }^{5}$ Zum Beispiel schreibt Johanides: „myslím, že film 322 režisér Hanák svojím spôsobom prešpekuloval a znehodnotil množstvom strihov" - allerdings stammt diese Einschätzung aus einem der späten Essays, die grundsätzlich herablassend-sentimental gefärbt sind (vgl. Pynsent 2005: 192).

6 Die Kapitel lauten „Cilka“, „Nevol’nost"“, „Zásluhy“, „Mám rada veci“, „Idem von“, „April showers ...", ,Vplyvy literatúry I“, „Ked’ na niečo myslíš, tak to nie je“, „Situácia I“, „In flagranti“, „Situácia II", „Spôsob útechy“, „Nerada čakám“, „Vplyvy literatúry II“ und „Resumé“. 
spielen eine Rolle - nicht nur durch eine Einlage auf der Bühne, sondern auch durch das häufige Herumtanzen und -tänzeln der Protagonisten. Schließlich werden auch die Medien Rundfunk und Fernsehen eingesetzt: während sich der Held rasiert, läuft im Radio das Morgenturnen, während er im Krankenhaus auf den Arzt wartet, hört er das Wunschkonzert, in einer Szene schauen sich strickende Frauen im Fernsehen eine Dokumentation über den Alltag von Textilarbeiterinnen an.

Der Verweis des Films auf seine Medialität, die Betonung von Form und Material, dessen künstlich-künstlerische Verwendung und Verfremdung bilden einen gewissen Kontrast zum authentischen Charakter des Films, der als großangelegte Montage konzipiert ist. Er wird deshalb nicht mehr als organisches Ganzes wahrgenommen, sondern verweist permanent auf seine Konstruiertheit; dies ist nach Peter Bürgers Theorie der Avantgarde Hauptprinzip und -merkmal der Avantgarde (vgl. Bürger 1993: 7 und $104 \mathrm{f}$.).

Bisher wurde Hanáks Film als Synthese der experimentellen Strömungen der sechziger Jahre - der authentisch-dokumentarischen wie der artifiziell-intellektuellen ${ }^{7}$ - beschrieben, das Wort Avantgarde wurde dabei wissentlich vermieden. Im Folgenden soll die Frage beantwortet werden, inwiefern „322“ zur Avantgarde oder Neo-Avantgarde gehört. Geht man von einem Avantgarde-Begriff aus, der den Bruch mit der Tradition, den experimentellen Kunstcharakter und die Provokation des Rezipienten in den Vordergrund rückt, dann kann der Film zumindest aus seinem historischen Kontext heraus als avantgardistisch gelten. Der radikale Bruch mit der dogmatischen, schematischen Kunst des Sozialistischen Realismus, wie sie in den fünfziger Jahren vorherrschte, ist offensichtlich.

Auch die Bezeichnung als Neo-Avantgarde ist zulässig, und das in zweierlei Hinsicht: einmal, weil der avantgardistische Anspruch wieder und neu gestellt wird, zum anderen aber auch, weil der Film ganz explizit an die historische Avantgarde anknüpft. Diese war in der tschechischen Literatur und Kunst der Zwischenkriegszeit verhältnismäßig stark ausgeprägt - man denke nur an den tschechischen Poetismus oder an die intensive Rezeption des Surrealismus, der mit dem „,nadrealizmus“ auch in der Slowakei bedeutend vertreten war. Nach der Unterdrückung im Faschismus und Kommunismus erlebte die Avantgarde in den sechziger Jahren eine Renaissance nicht nur in der wissenschaftlichen Beschäftigung, sondern auch im aktuellen Kunstschaffen.

Eine besonders starke Verbreitung hatte, wie bereits angedeutet, der Surrealismus. In Hanáks Film werden surrealistische Symbole und Motive breit gestreut: vom Schlüssel im goldenen Bilderrahmen über eine zerrissene Puppe ${ }^{8}$ bis hin zum

${ }^{7}$ Eine Klammer zwischen beiden Richtungen bilden auch Anfang und Ende des Films: im Vorspann deklamiert eine weibliche Stimme lateinische und griechische anatomische Begriffe wie „Corpus callosum“ oder „Hypothalamus“; am Ende stellt eine männliche Stimme lapidar fest: „to je lúka, to je jablko, to je strom“.

8 Roztrhané panenky war der Titel einer surrealistischen Gedichtsammlung von 1942. 
Körper und seinen Teilen als Objekt von Bildmontagen wie in einer Szene im Schlachthaus, wo Augen, Zungen und Kiefer von getöteten Tieren aufeinandergehäuft werden. ${ }^{9}$ Die Kunst der sechziger Jahre brachte eine Vielzahl von weiteren neo-avantgardistischen Strömungen hervor - oft in einer gewissen Parallelität zu den Vorgängern, zum Beispiel kehrt das expressionistische Entsetzen in der existentialistischen Bange wieder, der dadaistische Nonsens im Happening und in der absurden Kunst - die man ebenfalls in Hanáks Film finden kann.

Ján Johanides stand mit seiner existentialistisch gestimmten Novelle am Anfang der experimentellen Kunst der sechziger Jahre, Dušan Hanák befindet sich mit seinem Film an ihrem Ende. Die Produktion wurde zwar schon 1967 bewilligt, die Dreharbeiten begannen aber erst nach dem August 68 - in einer Zeit, als die künstlerische Avantgarde und Neo-Avantgarde zwar weitgehend institutionalisiert, aber durch die sich abzeichnende Normalisierung auch schon wieder bedroht waren. Insofern könnte man den Film, der 1970 aus dem Vertrieb genommen ${ }^{10}$ und erst nach 1989 wieder gespielt wurde, auch als unfreiwilligen Abgesang auf die sechziger Jahre verstehen.

Im Film sind, wie nachgewiesen wurde, zwei künstlerische Richtungen vereint, die sich auf den ersten Blick zu widersprechen scheinen: eine authentisch-dokumentarische und eine artifiziell-intellektuelle; zusammengeführt werden sie durch das Prinzip der Montage, das nach Bürger am Beginn der historischen Avantgarde stand. Dieses Prinzip wird letztlich auch der existentialistischen Grundidee der literarischen Vorlage gerecht, dass unser Dasein kein organisches Ganzes ist, sondern ein Zusammentreffen von Situationen, Ereignissen und Zufällen, deren Sinn sich nicht erschließt.

\section{L i t e rat u r}

Bürger 1993:

Felix 1997:

Holý 2003:

Marčok 2004:
Peter Bürger, Theorie der Avantgarde, Frankfurt am Main (9. Aufl.) Jozef Felix, Debut nekaždodenný, in: René Bílik, Eva Jenčíková, Jana Juráňová, Fedor Matejov, Jelena Pašteková und Zora Prušková (Hrsg.), Cítáme slovenskú literatúru II. 1956-1969, Bratislava, 185186

Jiří Holý, Geschichte der tschechischen Literatur des 20. Jahrhunderts, Wien

Vilém Marčok (Hrsg.), Dějiny slovenskej literatúry III. Cesty slovenskej literatúry druhou polovicou XX. storočia, Bratislava

${ }^{9}$ Gerade diese Szene erinnert an Georges Franjus Film „Le Sang des bêtes“ aus dem Jahr 1949 , in dem ebenfalls eine banale Geschichte mit drastischen Aufnahmen aus dem Schlachthaus zusammengeführt wird.

101969 war er nach Mannheim geschmuggelt und dort - ex aequo mit dem Film „Medium Cool“" des amerikanischen Regisseurs Haskell Wexler - mit dem Großen Preis der 18. Internationalen Filmwoche ausgezeichnet worden. 
Pynsent 2005: $\quad$ Robert P. Pynsent, Ján Johanides und die Genitalisten, in: Alfrun Kliems und Ludwig Richter (Hrsg.), Slowakische Kultur und Literatur im Selbst- und Fremdverständnis, Stuttgart, 179-212

A b stra ct: Dušan Hanák's Film "322" as a Neo-Avant-Garde Synthesis of the Experimental Art of the 1960s. In his film 322, Dušan Hanák joins together the authentically documentary and the artificially intellectual trends in the experimental art of the $1960 \mathrm{~s}$. The film should be considered as belonging to the neo-avant-garde, since, on the one hand, it rethinks the principle of the avant-garde (breaking with tradition, artistic experimentation, provocation of the recipient), and, on the other hand, it harks back to movements of the historical avant-garde (Dada, Surrealism) and employs avant-garde procedures (especially the montage technique). 322 is a synthesis and a culmination point of the neo-avant-garde art of the 1960s, whose further development was impaired after 1968.

K e yw ord s: avant-garde, neo-avant-garde, experimental art, Existentialism, 1960s, 322, Dušan Hanák, Ján Johanides, Slovak literature, Slovak film

Gertraude Zand

Institut für Slawistik der Universität Wien

Universitätscampus AAKH, Hof 3

Spitalgasse 2, 1090 Wien, Österreich

gertraude.zand@univie.ac.at 
